\title{
ON THE TREATMENT OF BOUNDARY ARTIFACTS IN IMAGE RESTORATION BY REFLECTION AND/OR ANTI-REFLECTION*
}

\author{
MARCO DONATELLI ${ }^{\dagger}$ AND STEFANO SERRA-CAPIZZANO $\ddagger$
}

\begin{abstract}
The abrupt boundary truncation of an image introduces artifacts in the restored image. For large image restoration with shift-invariant blurring, it is advisable to use Fast Fourier transform (FFT)-based procedures for reducing the computational effort. In this direction several techniques manipulate the observed image at the boundary or make some assumptions on the boundary of the true image, in such a way that FFT-based algorithms can be used. We compare the use of reflection with that of anti-reflection, in connection with the choice of the boundary conditions or for extending the observed image, both theoretically and numerically. Furthermore, we combine the two proposals. More precisely we apply anti-reflection, followed by reflection if necessary, to the observed image and we observe that the resulting restoration quality is increased with respect to the case of plain reflection.
\end{abstract}

Key words. Image deblurring, boundary conditions, fast transforms and matrix algebras.

AMS subject classifications. $65 \mathrm{~F} 22,65 \mathrm{~F} 15,65 \mathrm{Y} 20$.

1. Introduction. The blurred image is expressed as a function of an original scene that is larger than the field of view (FOV) of the blurred image, since pixels from the original scene outside the captured image window contribute to the pixels near the boundaries of the blurred observed image. Indeed the standard observation model can be expressed as

$$
g=A f_{o}+u,
$$

where $f_{o}$ and $g$, lexicographically ordered, are the true and observed images, and $u$ is the noise. The matrix $A$ represents a convolution of the true image $f_{o}$ with the point spread function (PSF) that we assume to be known and shift invariant. If the observed image is $n \times n$ and the PSF $m \times m$, then (1.1) implies that $f_{o}$ is $(n+m-1) \times(n+m-1)$ and that $A$ is a Toeplitz matrix of matrix size $n^{2} \times(n+m-1)^{2}$. This means that the linear system (1.1) is underdetermined. The goal is to recover $f_{o}$ only in the FOV, i.e., the image $f$ equal to the $n \times n$ middle part of $f_{o}$.

A well-established solution to both the problems of nonuniqueness and noise amplification is regularization. A classic approach is the Tikhonov regularization [10], which involves simultaneously minimizing the data error and a measure of the roughness of the solution. This leads to the linear system

$$
\left(A^{T} A+\mu I\right) f=A^{T} g,
$$

where $\mu>0$ is the regularization parameter that should be opportunely chosen and usually satisfies $\mu \ll 1$. In general the solution of the linear system (1.2) can be computationally expensive, since it is not automatic that an FFT-based algorithm can be applied directly. However an interesting approach is proposed in [8] when $m \ll n$. Indeed, for dealing with the rectangular matrix $A$ while using FFT-based

*This is a preprint of an article in the special volume "Matrix methods: theory, algorithms, applications" to be published by World Sci. Publishers

${ }^{\dagger}$ Dipartimento di Fisica e Matematica, Università dell'Insubria - Sede di Como, Via Valleggio 11, 22100 Como, Italy (marco.donatelli@uninsubria.it).

$\ddagger$ Dipartimento di Fisica e Matematica, Università dell’Insubria - Sede di Como, Via Valleggio 11, 22100 Como, Italy (stefano.serrac@uninsubria.it, serra@mail.dm.unipi.it). 
algorithms, it is necessary to resort to iterative methods [2], in which the main task is the application of FFT-based procedures for matrix-vector multiplication. Conversely, for employing FFT-based direct algorithms, the linear system to solve should have coefficient matrix diagonalizable by a suitable fast trigonometric transform, such as sine, cosine, $\omega$-Fourier $(|\omega|=1$ ), Hartley transforms (see e.g. [6]). This can be done modifying system (1.1) in order to obtain a square coefficient matrix. The first approach amounts in imposing boundary conditions (BCs) to $f_{o}$ an then computing a regularized solution of

$$
B f_{b}=g,
$$

where $B$ is $n^{2} \times n^{2}$, with a structure depending on the shift invariant kernel and on the type of BCs [5]. The second approach is to extend $g$ in some way to obtain $g_{e}$ of size $2 n \times 2 n$, and then regularizing

$$
C f_{e}=g_{e}
$$

where $C$ is the $(2 n)^{2} \times(2 n)^{2}$ Circulant matrix obtained by periodically completing $A$; here the restored image is the $n \times n$ part of $f_{e}$ corresponding to $g$ [1].

In this paper, we compare the two approaches in the case of reflective pad, i.e. the two proposals in [7] and [1]. We will also consider the use of reflection and antireflection in connection as possible choices for boundary conditions. The main results are the following:

- In the case of strongly symmetric (symmetric with respect to each axis independently) PSFs, the considered approaches produce comparable restorations in practical problems.

- Imposing anti-reflective boundary conditions leads to a better restoration quality with respect to the reflective boundary conditions, at least for moderate level of noise $[3,4,9]$. However a direct fast method is available only in the strong symmetric setting.

- To improve the results obtained by image extension as in (1.4), we use the ideas in [1], but instead of using reflection, we apply anti-reflection or antireflection followed by reflection. In this way we obtain a FFT-based algorithm also in the case of a generic PSF (non-necessarily symmetric), so overcoming the limitations in $[7,9]$ concerning the assumption of a strongly symmetric convolution kernel.

The paper is completed with numerical results to validate the proposals and the related analysis.

2. Reflection for image extension and BCs. In this section, we compare the reflection pad to extend $g$ and the imposition of reflective BCs. The proposal in [1] to extend $g$, is to form a new image $g_{e}$ of size $2 n \times 2 n$ as described in Fig. 1.1 (a). The observed image $g$ is at the top left quadrant, the top right quadrant is the mirror image of $g$ around the $y$ axis, and the bottom half is the mirror image of the top half around the $x$ axis. After that, the solution of the Tikhonov linear system is computed by circular convolution, because the coefficient matrix $C$ in (1.4) is Circulant. In [1] it is shown that, for 1-D images and symmetric PSFs, when the truncated image is locally stationary at its boundaries, this approach leads to smaller expected errors in the restored image with respect to apply directly the circular convolution to $g$. Indeed the circular convolution assumes a circular signal and, independently of $g, g_{e}$ is always a periodic image, hence it is reasonable to expect that it is obtained from a 


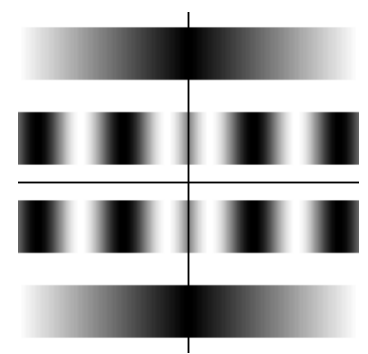

(a)

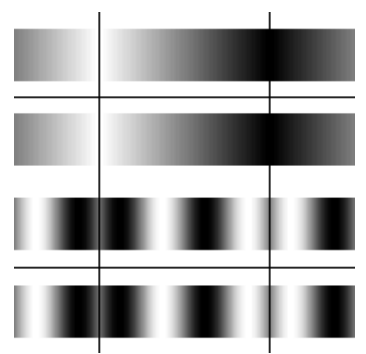

(b)

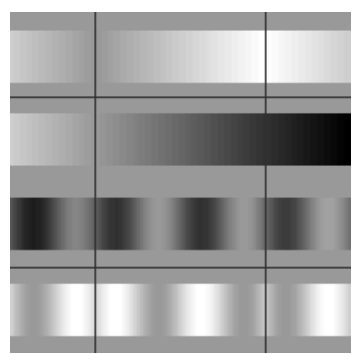

(c)

FIG. 1.1. (a) Full reflection of the left top quadrant on right and bottom. (b) Half reflection on each edge of the middle image. (c) Half anti-reflection on each edge of the middle image (scaled image). The edges of the images are emphasized by tiny vertical and horizontal lines.

periodic scene. This clearly reduces the boundary artifact in the restoration, also in the case of non-symmetric PSFs. We note that a reflection of size $n / 2$ with respect to each edge can be also used as in Fig. 1.1 (b), obtaining the same algorithm. Indeed the previous observation means only a translation of the period of the image of $n / 2$ in each variable.

The use of reflective or Neumann BCs implies that the true image outside the FOV is a reflection of the image inside the FOV. Therefore $f_{o}$ is assumed to be an extension by reflection of $f$ like in Fig. 1.1 (b). The reflection is done with respect to each edge with a bandwidth depending on the support of the PSF, since it is necessary each pixel at the boundary to be well defined. Imposing reflective BCs the square linear system has size $n^{2}$ and the matrix $B$ in (1.3) has a Toeplitz plus Hankel structure. More specifically, if the PSF is strongly symmetric, then $B$ can be diagonalized by the discrete cosine transform of type I (DCT-I) (two dimensional in the case of images).

Now we provide an algebraic formulation of the two approaches in the case of strongly symmetric PSFs and 1-D case. The latter will allow us to give a qualitative comparison of the solution computed from the two strategies applying the Tikhonov regularization to (1.3) and (1.4), respectively. Since the PSF is symmetric, we have $h=\left[h_{-q}, \ldots, h_{0}, \ldots, h_{q}\right]$ with $h_{-i}=h_{i}$ and $q=(m-1) / 2$. Let $\mathcal{T}_{k}=\left\{\phi_{\alpha}(x)=\right.$ $\left.\sum_{j=-k}^{k} \alpha_{j} \mathrm{e}^{\mathrm{i} j x}, \alpha_{-j}=\alpha_{j}\right\}$ be the set of even trigonometric polynomial of degree at most $k$, then the symbol

$$
\phi_{h}(x)=\sum_{j=-q}^{q} h_{j} \mathrm{e}^{\mathrm{i} j x}
$$

is such that $\phi_{h} \in \mathcal{T}_{q}$ and $q \leq(n-1) / 2$ for $m \leq n$.

Imposing reflective BCs, thanks to the symmetry of the PSF, in (1.3) $B=R_{n} D R_{n}^{T}$ where $R_{n}$ is the DCT-I matrix ( $R_{n}$ is real and orthogonal), $D=\operatorname{diag}(b)$ with $b=$ $R_{n}^{T}\left(B e_{1}\right) / R_{n}^{T} e_{1}$ (the division is component-wise) and $e_{1}$ is the first vector of the canonical base. Moreover, since $b_{i}=\phi_{h}(i \pi / n), i=0, \ldots, n-1, B$ can be expressed in terms of its symbol $\phi_{h}$ and it will be denoted by $B=\mathcal{R}_{n}\left(\phi_{h}\right)$ (see [7]). Therefore, using the Tikhonov regularization approach (1.2) for the linear system (1.3), we obtain

$$
f_{r}=R_{n} \operatorname{diag}\left(\frac{b}{b^{2}+\mu}\right) R_{n} g
$$


where the operations between vectors are intended component-wise. Setting $z=$ $b /\left(b^{2}+\mu\right)$ and by defining $p_{r} \in \mathcal{T}_{n-1}$ as the interpolating polynomial in the pairs $\left(\frac{i \pi}{n}, z_{i}\right)$ for $i=0, \ldots, n-1$, we find

$$
f_{r}=\mathcal{R}_{n}\left(p_{r}\right) g .
$$

For the other approach in (1.4), without loss of generality, let $\{1, \ldots, n\}$ be the FOV and $n$ be even. Hence, by reflecting $g=\left[g_{1}, \ldots, g_{n}\right]$ on both sides, we have

$$
g_{e}=\left[g_{n / 2}, \ldots, g_{2}, g_{1}, g_{1}, g_{2}, \ldots, g_{n}, g_{n}, g_{n-1}, \ldots, g_{n / 2+1}\right],
$$

that, as already observed, leads to the same proposal as in [1]. Defining

$$
P=\left[\begin{array}{c}
E_{l} \\
I \\
E_{r}
\end{array}\right]_{2 n \times n},
$$

$E_{l}=[J \mid 0]_{n / 2 \times n}, E_{r}=[0 \mid J]_{n / 2 \times n}$ and $J$ being the $n / 2 \times n / 2$ flip matrix with entries $[J]_{s, t}=1$ if $s+t=n+1$ and zero otherwise, we have $g_{e}=P g$. While $C=F_{2 n} \Lambda F_{2 n}^{H}$, where $F_{2 n}$ is the Fourier matrix of order $2 n$ and $\Lambda=\operatorname{diag}(c)$, with $c=$ $F_{2 n}^{H}\left(C e_{1}\right) / F_{2 n}^{H} e_{1}$. Since $c_{i}=\phi_{h}(2 \pi i / 2 n), i=0, \ldots, 2 n-1$, we denote $C=\mathcal{C}_{2 n}\left(\phi_{h}\right)$. Using the Tikhonov regularization (1.2) for the linear system (1.4), the restored signal of size $n$ is

$$
f_{c}=[0|I| 0]_{n \times 2 n} C_{2 n}\left(p_{c}\right) P g,
$$

where $I$ is the identity of order $n$ and, similarly to the reflective BCs case, $p_{c} \in \mathcal{T}_{2 n-1}$ is the interpolating polynomial in $\left(\frac{i \pi}{n}, v_{i}\right)$ for $i=0, \ldots, 2 n-1$ with $v=c /\left(c^{2}+\mu\right)$. We show that $p_{c} \in \mathcal{T}_{n}$ and it is the interpolating polynomial in $\left(\frac{i \pi}{n}, v_{i}\right)$ for $i=0, \ldots, n$, i.e., the points $\left(\frac{i \pi}{n}, v_{i}\right)$ for $i=n+1, \ldots, 2 n-1$ do not add any further information. The interpolation conditions are

$$
\begin{aligned}
p_{c}\left(\frac{i \pi}{n}\right) & =v_{i}, \quad i=0, \ldots, n, \\
p_{c}\left(\frac{(n+i) \pi}{n}\right) & =v_{n+i}, \quad i=1, \ldots, n-1 .
\end{aligned}
$$

From the trigonometric identity $\cos \left(\frac{(n+i) \pi}{n}\right)=\cos \left(\frac{(n-i) \pi}{n}\right)$, it follows $c_{n+i}=c_{n-i}$ that implies $v_{n+i}=v_{n-i}$ and $p_{c}\left(\frac{(n+i) \pi}{n}\right)=p_{c}\left(\frac{(n-i) \pi}{n}\right)$, for $i=1, \ldots, n-1$. Therefore, conditions $(2.8)$ can be written as $p_{c}\left(\frac{(n-i) \pi}{n}\right)=v_{n-i}$ for $i=1, \ldots, n-1$, that are a subset of (2.7). Moreover, $c_{i}=b_{i}$, and then $v_{i}=z_{i}$, for $i=0, \ldots, n-1$. Concluding, let $\Omega_{n}=\left\{\frac{i \pi}{n} \mid i=0, \ldots, n\right\}$ be the interpolation nodes forming a uniform grid on $[0, \pi]$ and let $\psi=\phi_{h} /\left(\phi_{h}^{2}+\mu\right)$, then

$$
\begin{array}{rll}
p_{c} \in \mathcal{T}_{n} & \text { interpolating } \psi \text { in } & \Omega_{n}, \\
p_{r} \in \mathcal{T}_{n-1} & \text { interpolating } \psi \text { in } & \Omega_{n} \backslash\{\pi\} .
\end{array}
$$

In order to compare $f_{r}$ with $f_{c}$, it remains to check whether $[0|I| 0] C_{2 n}\left(\phi_{\alpha}\right) P$ belongs 
to the DCT-I algebra. Let $\phi_{\alpha} \in \mathcal{T}_{\frac{n}{2}}$, then the $n \times 2 n$ matrix $T=[0|I| 0] C_{2 n}\left(\phi_{\alpha}\right)$ is

$$
T=\left[\begin{array}{cc|ccccc|cc}
\alpha_{-\frac{n}{2}} & \ldots & \alpha_{0} & \ldots & \alpha_{\frac{n}{2}} & & & & \\
& \ddots & \vdots & \ddots & \ddots & \ddots & & & \\
& & \alpha_{-\frac{n}{2}} & & & & \alpha_{\frac{n}{2}} & & \\
& & & \ddots & & & \vdots & \ddots & \\
& & & & \alpha_{-\frac{n}{2}} & \ldots & \alpha_{0} & \ldots & \alpha_{\frac{n}{2}}
\end{array}\right]
$$

and $T P=\mathcal{R}_{n}\left(\phi_{\alpha}\right)$. We note that $[0|I| 0] C_{2 n}\left(\phi_{\alpha}\right) P=\mathcal{R}_{n}\left(\phi_{\alpha}\right)$ holds only if $\phi_{\alpha} \in \mathcal{T}_{\frac{n}{2}}$. Therefore it can not be used in (2.6) since $p_{c} \in \mathcal{T}_{n}$, but it generally fails to belong to $\mathcal{T}_{\frac{n}{2}}$. However, from (2.3) and (2.6), it holds

$$
\begin{aligned}
f_{r}-f_{c} & =\left(\mathcal{R}_{n}\left(p_{r}\right)-[0|I| 0] C_{2 n}\left(p_{c}\right) P\right) g \\
& =\left(\mathcal{R}_{n}\left(p_{r}-\phi_{\alpha}\right)-[0|I| 0] C_{2 n}\left(p_{c}-\phi_{\alpha}\right) P\right) g,
\end{aligned}
$$

for $\phi_{\alpha} \in \mathcal{T}_{\frac{n}{2}}$. We take

$$
\phi_{\alpha}=\arg \min _{p \in \mathcal{T}_{\frac{n}{2}}}\|\psi-p\|_{\infty} .
$$

Therefore

$$
\begin{aligned}
\mathcal{C}_{2 n}\left(p_{c}-\phi_{\alpha}\right) & =\mathcal{C}_{2 n}\left(p_{c}-\psi+\psi-\phi_{\alpha}\right) \\
& =\mathcal{C}_{2 n}\left(r_{n}\right)+\mathcal{C}_{2 n}\left(a_{\frac{n}{2}}\right),
\end{aligned}
$$

where $r_{n}$ is the classical remainder in the trigonometric interpolation with $n+1$ equispaced nodes in $[0, \pi]$ with nodes belonging to $\Omega_{n}$, while $a_{\frac{n}{2}}$ is the sup-norm optimal remainder of degree $n / 2$. Similarly

$$
\mathcal{R}_{n}\left(p_{r}-\phi_{\alpha}\right)=\mathcal{R}_{n}\left(\tilde{r}_{n-1}\right)+\mathcal{R}_{n}\left(a_{\frac{n}{2}}\right),
$$

where $\tilde{r}_{n-1}$ is the remainder of the trigonometric interpolation with $n$ equispaced nodes in $[0, \pi]$ with nodes belonging to $\Omega_{n} \backslash\left\{x_{n}=\pi\right\}$. As a consequence, since the transforms associated with the circulant and the cosine algebras are unitary, it follows that the spectral norms $\left\|C_{n}(s)\right\|,\left\|\mathcal{R}_{n}(s)\right\|$ are bounded by the infinity norm of $s$. Moreover $\|P\|=\|[0|I| 0]\|=1$ and hence, by using (2.15)-(2.17) in (2.13), we find

$$
\begin{aligned}
\left\|f_{r}-f_{c}\right\| & \leq\left(\left\|\mathcal{R}_{n}\left(p_{r}-\phi_{\alpha}\right)\right\|+\left\|C_{2 n}\left(p_{c}-\phi_{\alpha}\right)\right\|\right)\|g\| \\
& \leq\left(\left\|r_{n}\right\|_{\infty}+\left\|\tilde{r}_{n-1}\right\|_{\infty}+2\left\|a_{n / 2}\right\|_{\infty}\right)\|g\| \\
& \leq 2\left(K n\left\|a_{n}\right\|_{\infty}+\left\|a_{n / 2}\right\|_{\infty}\right)\|g\|,
\end{aligned}
$$

with $K$ constant, where the latter inequality follows from the evaluation of the Lebesgue constants in the interpolation operators. In fact, after the change of variable $y=\cos (x)$, the operator behind $r_{n}$ is the interpolation on $[-1,1]$ with Chebyshev nodes of second type (the zeros of $\sin (n x) / \sin (x)$ ) plus the additional endpoints $\{ \pm 1\}$ : its Lebesgue constant is known to grow as $K \log (n)$. The other Lebesgue constant related to the operator behind $\tilde{r}_{n-1}$ is again related to the Chebyshev nodes of the second type plus only $y=1$ (i.e. $x=x_{0}=0$ ); in this case the associated Lebesgue constant is known to grow as $K n$. Since $\left\|a_{t}\right\|_{\infty}$ is exponentially converging to zero as $t$ tends to infinity (due to the $C^{\infty}$ regularity of $\psi$ ), it follows that $\left\|f_{r}-f_{c}\right\|$ is exponentially converging to zero as $n$ tends to infinity. As a consequence, the vectors $f_{r}$ 
and $f_{c}$ do not coincide in general, but their numerical difference is negligible already for moderate values of $n$.

Finally, when the PSF is not strongly symmetric, we notice that $B$ can not be diagonalized by DCT-I and it has only a Toeplitz plus Hankel structure. Therefore in general the linear system arising from Tikhonov regularization and reflective BCs can not be solved by a FFT-based algorithm. On the other hand, the other approach based on the extension of $g$ can be again applied without modifications.

3. Image extension by anti-reflection. The reflective pad is effective if the image is locally stationary at its boundaries, but it can still create significant artifacts if the image intensity has a large gradient at the boundary. Reflecting the image will create a cusp that is likely to be highly inconsistent with the original image, since the image beyond the boundary more than likely continues to change according to the gradient at the boundary rather than the negative of that gradient. According to this observation in [9], the author proposed to anti-reflect instead of to reflect the image at the boundary. The considered idea preserves the continuity of the normal derivative at the boundary without creating a cusp. In Fig. (1.1) (c) is shown how to extend an image by anti-reflection. We note a different scaling with respect to Fig. (1.1) (a) and Fig. (1.1) (b) since the anti-reflection produce value outside the original domain and the following visualization requires to scale the image.

We analyze in detail 1-D images. Imposing anti-reflective BCs the images $f=$ $\left[f_{1}, \ldots, f_{n}\right]$ is assumed to be extended as

$$
f_{1-j}=2 f_{1}-f_{j+1}, \quad f_{n+j}=2 f_{n}-f_{n-j},
$$

for $j=1,2, \ldots[9]$. Antireflective BCs usually provide restoration better than reflective BCs, also in practical 2-D applications, while, from a computational effort viewpoint, they share the same properties as the reflective BCs [3,4]. Indeed, when the PSF is strongly symmetric the matrix $B$ in (1.3) is essentially diagonalized by discrete sine transform of type III (DST-III), in the sense that the first and last equations are decoupled and the inner $(n-2) \times(n-2)$ block can be diagonalized by DST-III. Hence, several computations involving $B$, like Tikhonov regularization, can be done by FFT-based algorithms.

In the last case, PSF no strongly symmetric, the matrix $B$ is Toeplitz plus Hankel plus a rank two correction and the linear system arising from Tikhonov regularization can not be handled by simply invoking FFT-based algorithms. Therefore, when the PSF is not strongly symmetric, it could be useful to apply the anti-reflection pad to extend $g$ and regularizing (1.4). The extended image $g_{e}$ can be easily computed by $g_{e}=P g$, with $P$ defined in $(2.5)$ where now $E_{l}=[2 e|-J| 0]$ and $E_{r}=[0|-J| 2 e]$, $e=[1, \ldots, 1]^{T}$. We observe that in the case of a strongly symmetric PSF with the anti-reflective pad, differently from the reflective case, the two approaches (BCs on $f$ and extension of $g$ ) produce different restorations, usually of comparable quality: indeed the eigenvalues of $B$ are not a subset of the eigenvalues of $C$, as it happens for the reflective pad, even if they are defined on a uniform grid $\{i \pi /(n+1) \mid i=1, \ldots, n\}$ as well.

The main problem extending $g$ by anti-reflection is that $g_{e}$ is not periodic and then the model (1.4) could suffer from this. On the other hand the ringing effects are greatly reduced with respect to the application of the circulant de-convolution directly to $g$, since the boundaries are far away from the portion of the restored image, when compared with the circulant case. However, we can improve the model, and then the restoration, extending $g_{e}$ by reflection and obtaining a new periodic extended image 


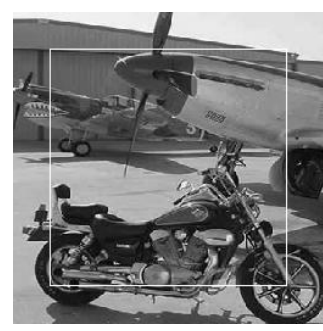

(a)

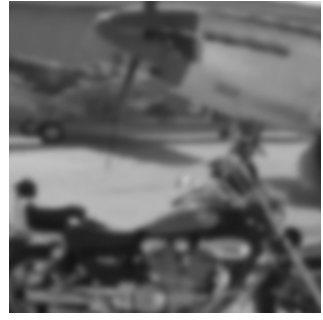

(b)

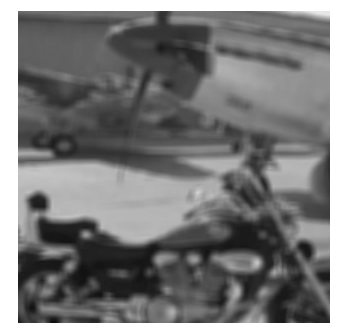

(c)

FIG. 4.1. (a) Original image where the box indicates observed region. (b) Gaussian blurred and noisy image. (c) Out of focus blurred and noisy image.

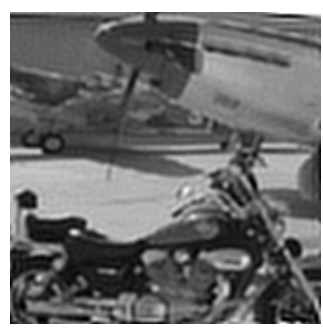

(a)

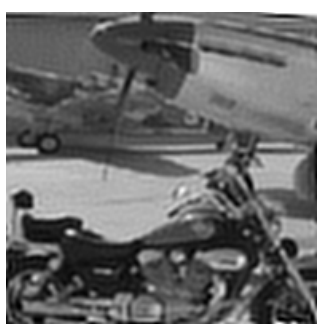

(b)

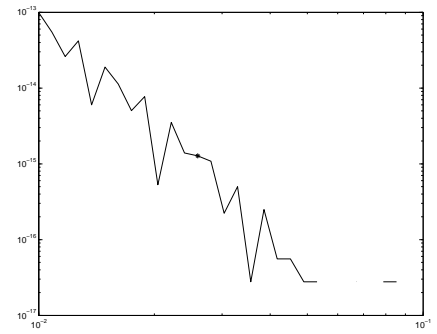

(c)

FIG. 4.2. Restorations of the image in Fig. 4.1 (b) (Gaussian blur): (a) restoration by reflective $B C s$, (b) restoration by reflective extension of the observed image, (c) loglog difference of the RREs for the two approaches ('*' corresponds to the optimal $\mu$ equal to 0.026 used in the restored images (a) and (b), absence of line means exactly zero value).

$g_{p}$ of size $4 n \times 4 n$. Clearly this further proposal leads to a moderate increase in the computational effort. In fact, as observed in [1], $g_{p}$ is real and symmetric and hence only the computation of the real part of a $2 \mathrm{D}$ FFT of size $2 n \times 2 n$ is required.

4. Numerical experiments. For the following experimentation we use Matlab 7.0. The blurred images are contaminated by a mild white Gaussian noise. The restorations are compared visually and the relative restoration error (RRE) is defined as $\|\hat{f}-f\|_{2} /\|f\|_{2}$, where $\hat{f}$ and $f$ are the restored and the true image respectively. For the Tikhonov regularization the parameter $\mu$ is chosen experimentally such that it minimizes the RRE, in a certain range of $\mu$.

The image in Fig 4.1 (a) was blurred with a Gaussian PSF (Fig. 4.1 (b)) and with an out of focus PSF (Fig. 4.1 (c)). The observed images are $n \times n$ with $n=195$. Since both the PSFs are strongly symmetric, we can compare the two approaches based on reflective $\mathrm{BCs}$ and reflective extension of the observed image respectively. The restored images and the absolute difference of the RREs for the two strategies in Fig. 4.2 and Fig. 4.3 validate the theoretical analysis given in Section 2. We note that both strategies reach the minimum RRE for the same value of $\mu$ and we observe that, around this minimum, the absolute difference of the RREs has the same order of the machine precision $\left(10^{-16}\right)$.

Now we consider the anti-reflective extension of the observed image described in Section 3 and we compare it only with the reflective extension in the case of a nonsymmetric PSF. Indeed, for strongly symmetric PSFs we have seen that the two approaches based on reflective BCs and reflective extension of the observed image are equivalent. Moreover, in the recent literature, it is widely documented a certain 


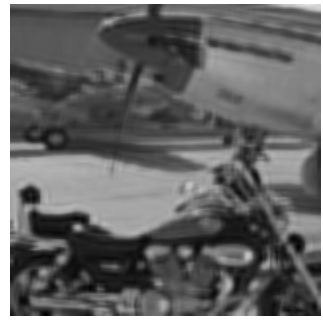

(a)

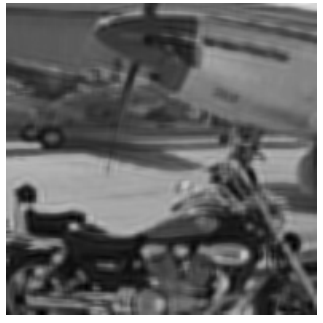

(b)

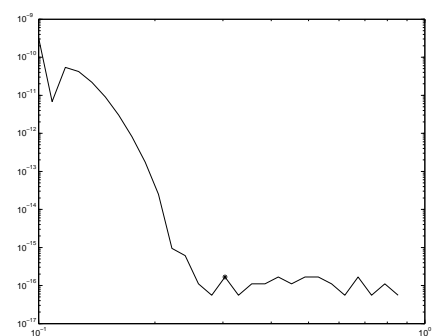

(c)

FIG. 4.3. Restorations of the image in Fig. 4.1 (c) (out of focus blur): (a) restoration by reflective BCs, (b) restoration by reflective extension of the observed image, (c) loglog difference of the RREs for the two approaches ('*' corresponds to the optimal $\mu$ equal to 0.304 used in the restored images (a) and (b)).

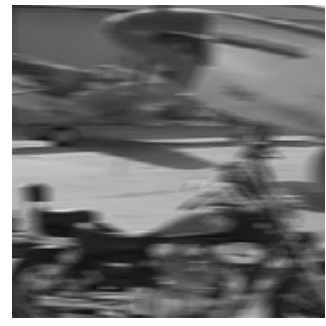

(a)

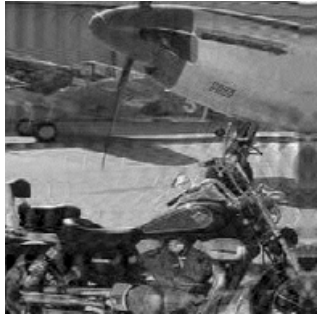

(b)

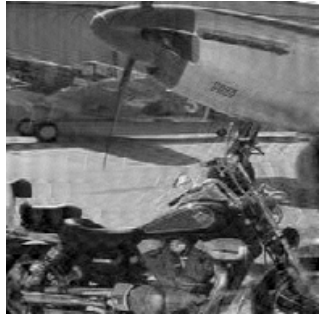

(c)

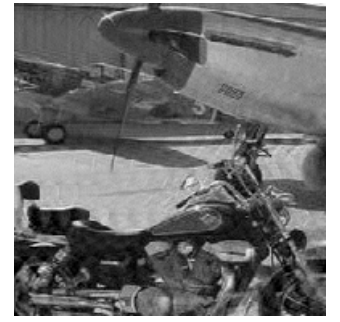

(d)

FIG. 4.4. (a) Moving blurred and noisy image. (b) Restoration by reflective extension $2 n \times$ $2 n(R R E=0.0932)$. (c) Restoration by anti-reflective extension $2 n \times 2 n(R R E=0.0807)$. (d) Restoration by anti-reflective extension and then reflective extension $4 n \times 4 n(R R E=0.0770)$.

supremacy of the anti-reflective BCs with respect to reflective BCs [3,4], for moderate levels of noise. On the other hand, when the PSF is not strongly symmetric the BC approach with the Tikhonov regularization leads to a linear system that can not be solved by FFT-based algorithms. Hence, in such case the only fast approach is which based on the extension of the observed image. According to the above comments we choose a PSF representing a motion along the $x$ axis. The original image is again that in Fig. 4.1 (a), while the blurred and noisy image is in Fig. 4.4 (a). In Fig. 4.4 (c) the restored image is obtained by anti-reflective extension that, also if the extended image is not periodic, is better than the restored image with reflective extension in Fig. 4.4 (b). The improvement is especially visible near the right edge, that is in the direction of the motion. If we want further improve the restoration, as described in Section 3 , we can extend by reflection the $2 n \times 2 n$ image obtained by the anti-refletive pad and then apply the circulant de-convolution to the new $4 n \times 4 n$ problem. Indeed, the restored image in Fig. 4.4 (d) is better than that in Fig. 4.4 (c). Moreover the last approach is more stable under perturbations of the parameter $\mu$, as shown in Fig. 4.5 by the plot of the RREs vs. $\mu$ for the considered approaches.

Acknowledgment. The work of the authors was partially supported by MUR, grant number 2006017542.

\section{REFERENCES}




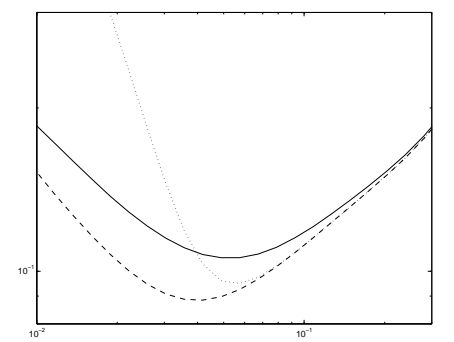

FIG. 4.5. Loglog RRE vs. $\mu$ for the test in Fig. 4.4 and the three approaches: - reflective extension, $\cdots$ anti-reflective extension $2 n \times 2 n,--$ anti-reflective extension and then reflective extension $4 n \times 4 n$.

[1] F. Aghdasi AND R. K. WARD, Reduction of boundary artifacts in image restoration, IEEE Trans. Image Process., 5 (1996), pp. 611-618.

[2] M. Bertero and P. BocCACCI, A simple method for the reduction of the boundary effects in the richardson-lucy approach to image deconvolution, Astron. Astrophys., 437 (2005), pp. 369-374.

[3] M. Christiansen And M. Hanke, Deblurring methods using antireflective boundary conditions. manuscript, 2006.

[4] M. Donatelli, C. Estatico, A. Martinelli, and S. Serra-Capizzano, Improved image deblurring with anti-reflective boundary conditions and re-blurring, Inverse Problems, 22 (2006), pp. 2035-2053.

[5] P. C. Hansen, J. G. Nagy, and D. P. O'Leary, Deblurring Images: Matrices, Spectra, and Filtering, SIAM, Philadelphia, PA, 2006.

6] T. Kailath And V. Olshevsky, Displacement structure approach to discrete-trigonometrictransform based preconditioners of g. strang type and t. chan type, Calcolo, 33 (1996), p. 191208.

[7] M. K. NG, R. H. Chan, And W. C. TANG, A fast algorithm for deblurring models with Neumann boundary conditions, SIAM J. Sci. Comput., 21 (1999), pp. 851-866.

[8] S. J. ReEves, Fast image restoration without boundary artifacts, IEEE Trans. Image Process., 14 (2005), pp. 1448-1453.

[9] S. Serra-Capizzano, A note on anti-reflective boundary conditions and fast deblurring models, SIAM J. Sci. Comput., 25 (2003), pp. 1307-1325.

[10] A. N. Tiknonov, Solution of incorrectly formulated problems and regularization method, Soviet Math. Dokl., 4 (1963), pp. 1035-1038. 